ORIGINAL

\title{
FACTORES DE RIESGO ASOCIADOS A LOS RESULTADOS POTENCIALMENTE INSATISFACTORIOS Y A LA MORTALIDAD DURANTE EL TRATAMIENTO ANTITUBERCULOSO EN ESPAÑA
}

\author{
Elena Rodríguez-Valín (1,2), Susana Villarrubia Enseñat (1), Oliva Díaz García (1) y Ele- \\ na Vanessa Martínez Sánchez (1,2).
}

(1) Centro Nacional de Epidemiología. Instituto de Salud Carlos III. Madrid

(2) CIBER de Epidemiología y Salud Pública (CIBERESP). Instituto de Salud Carlos III. Madrid.

Las autoras declaran no tener conflicto de intereses.

\section{RESUMEN}

Fundamentos: La curación del $85 \%$ de los casos de tuberculosis (TB) es uno de los retos de los programas de control de la enfermedad. El objetivo de este trabajo fue determinar los factores que influyen en los resultados insatisfactorios y la mortalidad durante el tratamiento antituberculoso en España.

Métodos: Estudio retrospectivo que incluyó 5.880 casos de TB declarados en 2012. Los resultados del tratamiento se clasificaron en: satisfactorios (RS: curación y tratamiento completo), potencialmente insatisfactorios (RPI: fracaso, traslado, abandono, tratamiento prolongado, desconocido) y fallecimiento. Se analizó la asociación del RPI y mortalidad con variables clínicas y epidemiológicas, mediante regresión logística. Se ajustaron dos modelos según el origen de los casos (español/extranjero).

Resultados: Los RS fueron $81 \%$ en españoles y $79 \%$ en extranjeros. Los españoles presentaron una mortalidad del $8 \%$ vs al $2 \%$ en los extranjeros) y menos traslados ( $2 \%$ vs $6 \%)$. A nivel multivariado los factores de riesgo asociados a RPI en españoles fueron la co-infección TB-VIH (OR 1,6 IC95\% 1,09-2,29) y el tratamiento previo (OR 2,4 IC95\% 1,67-3,53). En las personas extranjeras fueron la co-infección TB-VIH (OR 1,7 IC95\% $1,15-2,60)$, ser hombre (OR 1,4 IC95\% 1,11-1,83) y la localización pulmonar (OR 1,6 IC95\% 1,22-2,09). Los predictores de mortalidad en españoles fueron la co-infección TB-VIH (OR 2,7 IC95\% 1,63-4,54), ser hombre (OR 1,4 IC95\% 1,09-1,89), la localización pulmonar (OR 1,5 IC95\% 1,13-1,95) y la edad (OR 8,9 IC95\% 5,16-15,67 en mayores de 45 años). Y en extranjeros la co-infección TB-VIH (OR 3,2 IC95\% 1,53-6,76), ser hombre (OR 2,2 IC95\% 1,01-4,60) y la edad (OR 3,4 IC95\% 1,81-6,48 en mayores de 45 años).

Conclusiones: El porcentaje de resultados satisfactorios del tratamiento antituberculosos en España no alcanza los objetivos internacionales, especialmente en las personas extranjeras. Para disminuir los resultados insatisfactorios y la mortalidad es necesario mejorar el manejo de los sujetos coinfectados TB-VIH, los casos previamente tratados, las localizaciones pulmonares, a los hombres y a las personas de edad avanzada.

Palabras clave: Tuberculosis. Resultados del tratamiento. Mortalidad. Vigilancia epidemiológica. Inmigración.

\section{Correspondencia}

Elena Rodríguez-Valín

Centro Nacional de Epidemiología. Instituto de Salud Carlos III.

C/ Monforte de Lemos, 5. 28029-Madrid.

Teléfono: 918222614

Fax: 913877816

Correo electrónico: ervalin@isciii.es

DOI:

\section{ABSTRACT \\ Risk Factors for Potentially Unsuccessful Results and Mortality during Duberculosis Treatment in Spain}

Background: Successful result of treatment in $85 \%$ of cases is the target of a Tuberculosis (TB) Control Programme. The aim of this study is to determine the risk factors for unsuccessful completion of treatment and deaths of TB cases in Spain.

Methods: Data from the National Surveillance Epidemiological Network. Retrospective study including 5,880 TB cases reported in 2012 . Outcomes were classified as: successful (S: cured and treatment completed), potentially unsuccessful (PU: failed, transferred, defaulted, still on treatment and unknown) and deaths. Logistic regression analysis was used to explore the association between epidemiological and clinical factors with PU and deaths as outcome variables. Two different models for nationals and foreigners were adjusted.

Results: Successful outcome was $81 \%$ in nationals and $79 \%$ in foreigners. Spanish TB cases had higher mortality rates than foreigners ( $8 \% \mathrm{vs}$. $2 \%$ ), and lower percentage of transfers ( $2 \%$ vs. $6 \%$ ). At multivariate level the risk factors for PU in nationals were: HIV coinfection (OR 1.6 CI95\% 1.09-2.29) and previous treatment (OR 2.4 CI95\% $1.67-3.53$ ); and HIV coinfection (OR 1.7 CI95\% 1.15-2.60), male sex (OR 1.4 CI95\% 1.11-1.83) and pulmonary TB (OR 1.6 CI95\% 1.22-2.09) in foreigners. Risk factors for death in nationals were: HIV coinfection (OR 2.7 CI95\% 1.63-4.54), male sex (OR 1.4 CI95\% 1.09-1.89), pulmonary TB (OR 1.5 CI95\% 1.13-1.95) and an increasing risk with age (OR 8.9 CI95\% 5.16-15.67 in over 45 years group.).; and HIV coinfection (OR 3.2 CI95\% 1.53-6.76), male sex (OR 2.2 C195\% 1.01-4.60) and older age(OR 3.4 CI95\% 1.81-6.48 in over 45 years group.) in foreigners.

Conclusion: The rate of successful treatment in Spain does not meet the international target, especially in foreigners. To reduce unsuccessful treatment and deaths it is necessary to improve management in TB-HIV co-infected cases, and those with previous treatment story, pulmonary TB, males and older age.

Key words: Tuberculosis. Treatment outcome. Mortality. Epidemiological surveillance. Immigrants. 


\section{INTRODUCCIÓN}

El principal objetivo de los programas de Control de la tuberculosis (TB) ha sido durante décadas la identificación precoz y el tratamiento adecuado de los casos nuevos, para reducir el riesgo de exposición en los demás miembros de la comunidad, disminuyendo la incidencia y prevalencia de la enfermedad. En los países de baja incidencia, que aspiran al objetivo de la eliminación, esta medida se complementa con el estudio de los contactos para cada caso diagnosticado y la aplicación de terapia preventiva o su seguimiento para interrumpir la cadena de transmisión, junto con el diseño de estrategias específicas dirigidas a los grupos más vulnerables $^{1,2}$.

España es considerada un país de baja incidencia de tuberculosis (TB), según los criterios del European Centre for Disease Prevention and Control (ECDC), que incluye en este grupo a los países con incidencia inferior a $20 \mathrm{ca}-$ sos/100.000 habitantes ${ }^{3}$. En el año 2012, la incidencia de todas las formas de tuberculosis en España fue de 13 casos/100.000 habitantes, con un descenso medio anual de $-8 \%$ en el periodo 2008-2012,5. En el 2013 la incidencia siguió descendiendo, siendo de 11,9 casos/100.000 habitantes ${ }^{6}$.

La monitorización de los resultados del tratamiento en los países de la Unión Europea/ Área Económica Europea (UE/AEE) se basa en las recomendaciones del Grupo de Trabajo conjunto de la Organización Mundial de la Salud/Unión Internacional contra la Tuberculosis y las Enfermedades Respiratorias (OMS/ UICTER), establecido en 1997. Su objetivo fue establecer las definiciones y procedimientos comunes para el seguimiento terapéutico de las personas con tuberculosis, permitiendo su comparabilidad internacional y la evaluación de la eficacia de los Programas de TB en los países? ${ }^{7}$ Los objetivos globales de la OMS eran conseguir al menos un porcentaje de resultados satisfactorios del $85 \%$ de los casos, considerando aceptable un porcentaje de fallecimientos del $5 \%$ y por tanto un $10 \%$ máximo de resultados potencialmente insatisfactorios. Los porcentajes se suelen calcular sobre los casos nuevos de localización pulmonar confirmados por cultivo, que son los prioritarios desde el punto de vista de la transmisión.

A pesar de los avances realizados desde entonces en materia de prevención y control, en la UE/AEE todavía no se han alcanzado estos objetivos. En 2012 hubo un porcentaje global de resultados satisfactorios de tratamiento del $73 \%$, lo que se debe no sólo a las diferencias en la situación epidemiológica de la TB entre los países sino a deficiencias en los sistemas de vigilancia y notificación ${ }^{8,9}$.

En España, distintos estudios de investigación han mostrado un porcentaje de resultados satisfactorios comprendido entre el 70 y el $89 \%$, si bien no son extrapolables al total de la población ${ }^{10-12}$. Los datos procedentes de los Programas de tuberculosis de distintas comunidades autónomas (CCAA) muestran unos porcentajes cercanos al 90\%, si bien hay que señalar que, como en todos los sistemas de información que se basan en la declaración de casos, puede haber problemas de subnotificación así como de casos de los que no se conocen los resultados de tratamiento ${ }^{13-15}$.

El objetivo de este estudio fue describir los resultados del tratamiento antituberculoso en España así como determinar los factores que influyen en los resultados potencialmente insatisfactorios y en la mortalidad.

\section{MATERIAL Y MÉTODO}

Para la realización de este estudio retrospectivo se incluyeron los casos de TB diagnosticados en el año 2012 y notificados a la Red Nacional de Vigilancia Epidemiológica (RENAVE). La definición de caso de TB fue la recogida en los protocolos de vigilancia ${ }^{16}$, considerándose caso a todo paciente con $\mathrm{TB}$ pulmonar o extrapulmonar diagnosticado en el año de estudio y que cumplía los siguientes criterios: clínica correspondiente a TB de cualquier localización y prescripción de tratamiento (caso no confirmado bacteriológicamente), o si adicionalmente a los criterios clínicos tenía 
microscopia positiva o bien que presentara un aislamiento por cultivo de un microorganismo del complejo $M$. tuberculosis (caso confirmado bacteriológicamente). En el protocolo de vigilancia se establece que todos los casos de TB diagnosticados durante el año confirmados bacteriológicamente o no, deben notificarse a la RENAVE, y los resultados de tratamiento se deben actualizar el año siguiente de la notificación. Las CCAA notifican los datos al Centro Nacional de Epidemiología (CNE) vía electrónica mediante ficheros en los que los datos personales se han anonimizado y mantenido de forma confidencial en origen, por lo que no es posible su identificación en el nivel nacional.

El estudio incluyó 5.880 casos de TB declarados a la RENAVE, diagnosticados en el año 2012 y con información sobre resultados del tratamiento antituberculoso (actualizada a diciembre de 2014). Las categorías de resultados de tratamiento a los 12 meses de su inicio, según el protocolo de vigilancia ${ }^{17}$, fueron las siguientes:

Curación: paciente que completó el tratamiento y además presentaba al menos un cultivo o baciloscopia negativas en muestras tomadas al final del mismo y, como mínimo, en otra ocasión previa.

Tratamiento completo: paciente que completó el tratamiento y no cumplía criterios para ser clasificado como curación o fracaso terapéutico.

Fracaso terapéutico: paciente que cinco meses después de haber iniciado el tratamiento y habiéndolo realizado correctamente sigue con cultivo o baciloscopia positivos.

Traslado: paciente que cambió su residencia y por ello fue transferido a otro sistema de registro y cuyos resultados terapéuticos eran desconocidos.

Abandono: paciente que interrumpió el tratamiento durante dos o más meses, sin que se debiera a una decisión facultativa.
Fallecimiento: paciente fallecido por cualquier causa durante el curso del tratamiento. También incluyó a los diagnosticados post mortem.

Otro, no evaluado: paciente que continuó en tratamiento a los 12 meses de haberlo iniciado debido a que se le prolongó como consecuencia de efectos secundarios/complicaciones o se planificó desde el inicio con una duración mayor de 12 meses (incluyó pacientes poliresistentes) o que continuó en tratamiento sin saberse la causa.

Desconocido: paciente perdido en el seguimiento antes de finalizar su tratamiento, excepto en el caso de que se trate de un traslado, o paciente del que se desconocen los resultados.

Se consideraron casos nuevos las personas que nunca habían recibido tratamiento antituberculoso o que lo habían recibido durante menos de un mes. Y casos tratados previamente a los pacientes que recibieron tratamiento al menos durante un mes (excluyendo tratamiento frente a la infección tuberculosa latente).

A efectos del análisis, los casos se agruparon en tres categorías: resultados satisfactorios (RS: curación y tratamiento completo), resultados potencialmente insatisfactorios (RPI: fracaso, traslado, abandono, otros y desconocidos) y fallecimiento. Se realizó un análisis descriptivo de los casos según distintas variables recogidas en la declaración (sexo, edad, localización anatómica de la tuberculosis, origen, tratamiento previo, confirmación por cultivo y serología del VIH) para cada una de las categorías (RS, RPI y mortalidad). La edad como variable continua se caracterizó por su media y desviación estándar. Para el análisis se distribuyeron los casos en cuatro grupos de edad: 0-14 años, 15-44, 45-64 y mayores de 65.

Posteriormente se estudió la asociación de las variables recogidas en la declaración con la mortalidad y con los RPI como variables dependientes, calculándose las Odds Ratio crudas (ORc) mediante un análisis bivariado y las ajustadas (ORa) por regresión logística. 
Las variables con significación estadística $(\mathrm{p}<0,05)$ en el análisis bivariado y las que sin ser significativas se consideraron de interés epidemiológico se incluyeron en el modelo de regresión logística. Se elaboró un modelo de regresión hacia atrás (stepwise) en el que se incluyeron todas las variables y se fueron eliminando aquellas que no fueron estadísticamente significativas $(\mathrm{p}>0,05)$. Se calculó la bondad de ajuste del modelo por el método de Hosmer y Lemeshow. El análisis se realizó con el paquete estadístico Stata 13.1. Para el análisis de la mortalidad se incluyeron todos los casos y para el análisis del RPI se excluyeron los fallecimientos.

\section{RESULTADOS}

De los 5.880 casos de TB con información sobre resultados de tratamiento, 4.703 $(80 \%)$ presentaron un resultado satisfactorio, de los que 2.365 se declararon curados y 2.338 con tratamiento completo. En cuanto a los RPI, $797(13,5 \%)$ casos presentaron RPI y $380(6,5 \%)$ fallecieron por cualquier causa durante el tratamiento. Los 797 casos con RPI se distribuyeron de la siguiente manera: 8 fracasos terapéuticos, 182 traslados, 79 abandonos, 60 "otros" y 468 desconocidos. Si consideramos solamente los casos nuevos pulmonares confirmados por cultivo (2.949 casos), los porcentajes de RS, RPI y muertes fueron $82 \%, 11 \%$ y $7 \%$ respectivamente.

Las características de los casos en las tres categorías (RS, RPI y fallecimiento) se muestran en la tabla 1. El porcentaje de hombres (63\%) fue superior al de mujeres, especialmente entre los casos fallecidos durante el tratamiento (75\%). El 71\% de los casos presentaron localización pulmonar. Respecto al origen de los casos, la distribución es muy diferente en las tres categorías. En todas, el porcentaje de casos españoles es superior al de extranjeros, destacando el grupo de fallecimientos, en los que el $87 \%$ son españoles.

\section{Figura 1}

Resultados del tratamiento antituberculoso en personas españolas y extranjeras para el total de casos y excluyendo los fallecimientos. España, 2012.

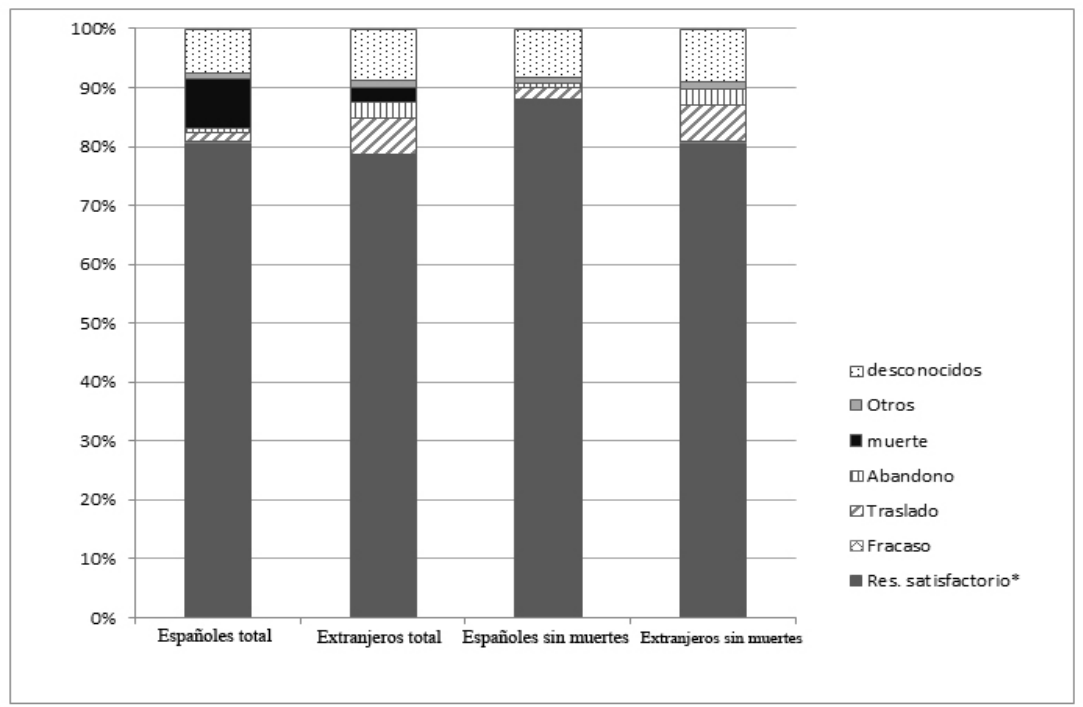

Fuente: Red Nacional de Vigilancia Epidemiológica. * Resultados satisfactorios: curación + tratamiento completo 


\begin{tabular}{|c|c|c|c|c|c|c|c|c|c|}
\hline \multicolumn{10}{|c|}{$\begin{array}{c}\text { Tabla } 1 \\
\text { Características de los casos de tuberculosis según los resultados de tratamiento } \\
\text { y las variables de estudio. España, } 2012\end{array}$} \\
\hline \multirow{2}{*}{\multicolumn{2}{|c|}{ Variables }} & \multicolumn{2}{|c|}{$\begin{array}{l}\text { Resultados } \\
\text { Potencialmente } \\
\text { satisfactorios } \\
\text { (excluye muerte) }\end{array}$} & \multicolumn{2}{|c|}{$\begin{array}{l}\text { Resultados } \\
\text { satisfactorios }\end{array}$} & \multicolumn{2}{|c|}{ Fallecimientos } & \multicolumn{2}{|c|}{ Total } \\
\hline & & $\mathrm{n}$ & $\%$ & $\mathrm{n}$ & $\%$ & $\mathrm{n}$ & $\%$ & $\mathrm{n}$ & $\%$ \\
\hline \multirow{4}{*}{ Edad } & $0-14$ & 31 & 3,89 & 313 & 6,66 & 0 & 0,00 & 344 & 5,85 \\
\hline & $15-44$ & 451 & 56,59 & 2398 & 50,99 & 40 & 10,53 & 2889 & 49,13 \\
\hline & $45-64$ & 183 & 22,96 & 1120 & 23,81 & 104 & 27,37 & 1407 & 23,93 \\
\hline & $>65$ & 132 & 16,56 & 871 & 18,52 & 236 & 62,11 & 1239 & 21,07 \\
\hline \multirow{2}{*}{ Sexo } & Mujer & 262 & 32,87 & 1832 & 38,95 & 96 & 25,26 & 2190 & 37,24 \\
\hline & Hombre & 535 & 67,13 & 2871 & 61,05 & 284 & 74,74 & 3690 & 62,76 \\
\hline \multirow{2}{*}{ Origen } & Nacional & 442 & 55,46 & 3219 & 68,45 & 331 & 87,11 & 3992 & 67,89 \\
\hline & Extranjero & 355 & 44,54 & 1484 & 31,55 & 49 & 12,89 & 1888 & 32,11 \\
\hline \multirow{3}{*}{$\begin{array}{l}\text { Tratamiento } \\
\text { previo }\end{array}$} & No & 655 & 82,18 & 4324 & 91,94 & 332 & 87,37 & 5311 & 90,32 \\
\hline & Sí & 71 & 8,91 & 225 & 4,78 & 27 & 7,11 & 323 & 5,49 \\
\hline & Desconocido & 71 & 8,91 & 154 & 3,27 & 21 & 5,53 & 246 & 4,18 \\
\hline \multirow{3}{*}{ VIH } & Negativo & 507 & 63,61 & 3143 & 66,83 & 239 & 62,89 & 3889 & 66,14 \\
\hline & Positivo & 79 & 9,91 & 272 & 5,78 & 31 & 8,16 & 382 & 6,50 \\
\hline & Desconocido & 211 & 26,47 & 1288 & 27,39 & 110 & 28,95 & 1609 & 27,36 \\
\hline \multirow{2}{*}{ Localización } & Extrapulmonar & 231 & 28,98 & 1373 & 29,19 & 101 & 26,58 & 1705 & 29,00 \\
\hline & Pulmonar & 566 & 71,02 & 3330 & 70,81 & 279 & 73,42 & 4175 & 71,00 \\
\hline \multirow{3}{*}{ Cultivo } & Negativo & 133 & 16,69 & 948 & 20,16 & 54 & 14,21 & 1135 & 19,30 \\
\hline & Positivo & 521 & 65,37 & 3238 & 68,85 & 287 & 75,53 & 4046 & 68,81 \\
\hline & Desconocido & 143 & 17,94 & 517 & 10,99 & 39 & 10,26 & 699 & 11,89 \\
\hline \multicolumn{2}{|r|}{ Total } & 797 & 100,00 & 4703 & 100,00 & 380 & 100,00 & 5880 & 100,00 \\
\hline
\end{tabular}

El porcentaje de casos tratados previamente oscila entre el 5\% de los RS y el 9\% de los RPI. El 68,8\% de los casos fueron confirmados por cultivo, oscilando entre el $65 \%$ en los RPI y $75 \%$ en los fallecidos. El $6 \%$ del total de los casos fueron VIH positivos, si bien los RPI fueron los que tuvieron un mayor porcentaje $(10 \%)$. Finalmente, respecto a la edad, los casos con RPI y RS fueron más jóvenes (56,6\% y 51\% tenían entre 15 y 44 años, respectivamente), mientras que en los fallecidos el $62 \%$ tenían más de 65 años (edad media de $68,80+17,23$ años en fallecidos frente a 43,19+19,81 y 43,38+21 en los RPI y RS respectivamente). También hay que señalar que en niños no hubo ningún fallecimiento $\mathrm{y}$ que el número de resultados satisfactorios fue muy elevado $(90 \%)$.
Durante la realización de los análisis para ver la relación de las variables independientes con el RPI o la mortalidad se observó que el origen de los casos (España/extranjero) era un modificador del efecto de las demás variables sobre el resultado del tratamiento antituberculoso, por lo que se decidió estudiar separadamente los casos nacidos en España y los nacidos fuera de España.

Las categorías de finalización del tratamiento en españoles y extranjeros respecto al total de casos y excluyendo los fallecimientos se muestran en la figura 1. Para el total de casos, el porcentaje de RS (curación más tratamiento completo) fue en los nacidos en España del $81 \%$ y en los nacidos en otros países del 79\%. En cuanto a los RPI, el porcentaje de desconocidos fue del $7,6 \%$ en españoles 


\section{Tabla 2}

Variables asociadas a los resultados potencialmente insatisfactorios (RPI) del tratamiento antituberculoso según el origen de los casos. Resultados del análisis bivariado y multivariado. España, 2012

\begin{tabular}{|c|c|c|c|c|c|c|c|}
\hline \multirow{2}{*}{\multicolumn{2}{|c|}{ Variables }} & \multicolumn{3}{|c|}{ Nacidos en España } & \multicolumn{3}{|c|}{ Nacidos fuera de España } \\
\hline & & \multirow{2}{*}{ n RPI (\%) } & \multirow{2}{*}{$\begin{array}{c}\text { Bivariado } \\
\text { ORc (IC 95\%) }\end{array}$} & \multirow{2}{*}{$\begin{array}{c}\text { Multivariado } \\
\text { ORa (IC 95\%) } \\
\end{array}$} & n RPI (\%) & \multirow{2}{*}{$\begin{array}{c}\text { Bivariado } \\
\text { ORc (IC 95\%) }\end{array}$} & \multirow{2}{*}{\begin{tabular}{|c|} 
Multivariado \\
ORa (IC 95\%) \\
\end{tabular}} \\
\hline \multirow{5}{*}{ Edad } & & & & & & & \\
\hline & 0-14 (ref.) & $52(9,70)$ & & & $70(19,94)$ & & \\
\hline & $15-44$ & $135(11,82)$ & $0,95(0,77-1,17)$ & ns & $225(19,33)$ & $1,14(0,85-1,53)$ & ns \\
\hline & $45-64$ & $135(13,13)$ & $1,14(0,92-1,42)$ & ns & $48(17,45)$ & $0,86(0,62-1,21)$ & ns \\
\hline & $>65$ & $120(12,58)$ & $1,06(0,85-1,33)$ & ns & $12(24,49)$ & $1,37(0,70-2,65)$ & ns \\
\hline \multirow{2}{*}{ Sexo } & Mujer (ref.) & $144(10,77)$ & & & $118(15,59)$ & & \\
\hline & Hombre & $298(12,82)$ & $1,22(0,98-1,50)$ & ns & $237(21,90)$ & $1,52(1,19-1,94) *$ & $1,42(1,11-1,83)^{*}$ \\
\hline \multirow{3}{*}{ Tratamiento previo } & No (ref.) & $374(11,14)$ & & & $281(17,33)$ & & \\
\hline & Sí & $43(23,89)$ & $2,42(1,69-3,47)^{*}$ & $2,44(1,67-3,53)^{*}$ & $28(24,14)$ & $1,36(0,87-2,11)$ & ns \\
\hline & Desconocido & $25(20,33)$ & $1,91(1,21-2,99)^{*}$ & $2,15(1,36-3,39)^{*}$ & $46(45,10)$ & $3,79(2,51-5,74)^{*}$ & $3,81(2,52-5,77)^{*}$ \\
\hline \multirow{3}{*}{ VIH } & Negativo (ref.) & $274(11,58)$ & & & $233(18,15)$ & & \\
\hline & Positivo & $41(18,81)$ & $1,76(1,23-2,51)^{*}$ & $1,58(1,09-2,29)^{*}$ & $38(28,57)$ & $1,75(1,18-2,60)^{*}$ & $1,73(1,15-2,60)^{*}$ \\
\hline & desconocido & $127(11,79)$ & $0,96(0,77-1,19)$ & $\mathrm{ns}$ & $84(19,91)$ & $1,05(0,79-1,38)$ & $\mathrm{ns}$ \\
\hline \multirow[t]{2}{*}{ Localización } & $\begin{array}{l}\text { Extrapulmonar } \\
\text { (ref.) }\end{array}$ & $142(14,46)$ & & & $89(14,81)$ & & \\
\hline & Pulmonar & $300(11,29)$ & $0,77(0,62-0,96)^{*}$ & ns & $266(21,49)$ & $1,57(1,21-2,05) *$ & $1,60(1,22-2,09)^{*}$ \\
\hline \multirow{3}{*}{ Cultivo } & Negativo (ref.) & $78(10,79)$ & & & $55(15,36)$ & & \\
\hline & Positivo & $262(10,66)$ & $0,68(0,55-0,83)^{*}$ & ns & $259(19,89)$ & $1,14(0,88-1,48)$ & ns \\
\hline & Desconocido & $102(21,21)$ & $2,25(1,75-2,88)^{*}$ & $2,37(1,85-3,04)^{*}$ & $41(22,91)$ & $1,27(0,88-1,84)$ & $\mathrm{ns}$ \\
\hline
\end{tabular}

* $\mathrm{p}<0,05$. ORc: Odds Ratio cruda, ORa: Odds Ratio ajustada, IC: Intervalo de confianza. n RPI: número de casos con Resultados Potencialmente Insatisfactorios. ref.: categoría de referencia Fuente. Red Nacional de Vigilancia Epidemiológica 


\begin{tabular}{|c|c|c|c|c|c|c|c|}
\hline & $\begin{array}{r}\text { Variables a } \\
\text { gún el origen de } 1\end{array}$ & $\begin{array}{l}\text { ciadas a la m } \\
\text { casos. Resul }\end{array}$ & $\begin{array}{r}\text { Tabla } 3 \\
\text { ortalidad dura } \\
\text { tados del anális }\end{array}$ & $\begin{array}{l}\text { nte el tratamiento } \\
\text { is bivariado y mu }\end{array}$ & $\begin{array}{l}\text { antituberc } \\
\text { Itivariado. }\end{array}$ & $\begin{array}{l}\text { Uuloso } \\
\text { España, } 2012\end{array}$ & \\
\hline & & & Nacidos en Espa & & & Nacidos fuera de $\mathrm{E}$ & ispaña \\
\hline & & Número & Bivariado & Multivariado & Número & Bivariado & Multivariado \\
\hline Variables & & $\begin{array}{c}\mathrm{de} \\
\text { defunciones } \\
(\%)\end{array}$ & ORc (IC 95\%) & ORa (IC 95\%) & $\begin{array}{c}\text { de } \\
\text { defunciones } \\
(\%)\end{array}$ & ORc (IC 95\%) & ORa (IC 95\%) \\
\hline & 0-14 (ref.) & $1(0,19)$ & & & $3(0,85)$ & & \\
\hline Fdad & $15-44$ & $14(1,21)$ & $0,07(0,04-0,13)^{*}$ & ns & $22(1,85)$ & $0,27(0,15-0,49) *$ & $\mathrm{~ns}$ \\
\hline Edad & $45-64$ & $87(7,80)$ & $0,91(0,71-1,18)$ & $8,99(5,16-15,67)^{*}$ & $17(5,82)$ & $3,02(1,65-5,53) *$ & $3,43(1,81-6,48)^{*}$ \\
\hline & $>65$ & $229(19,36)$ & $6,37(4,94-8,20)^{*}$ & $29,49(17,28-50,35)^{*}$ & $7(12,50)$ & $6,09(2,59-14,3) *$ & $9,25(3,75-22,82)^{*}$ \\
\hline Soyo & Mujer (ref.) & $86(6,04)$ & & & $10(1,3)$ & & \\
\hline Sexo & Hombre & $245(9,56)$ & $1,64(1,27-2,11)^{*}$ & $1,44(1,09-1,89)^{*}$ & $39(3,5)$ & $2,73(1,35-5,51)^{*}$ & $2,25(1,01-4,60)^{*}$ \\
\hline & No (ref.) & $291(7,97)$ & & & $41(2,47)$ & & \\
\hline Tratamiento previo & Sí & $23(11,33)$ & $1,44(0,92-2,26)$ & ns & $4(3,33)$ & $1,32(0,47-3,73)$ & ns \\
\hline & Desconocido & $17(12,14)$ & $1,56(0,92-2,26)$ & ns & $4(3,77)$ & $1,51(0,53-4,29)$ & ns \\
\hline & Negativo (ref.) & $206(8,01)$ & & & $33(2,51)$ & & \\
\hline VIH & Positivo & $21(8,79)$ & $1,07(0,67-1,69)$ & $2,73(1,63-4,54)^{*}$ & $10(6,99)$ & $3,28(1,60-6,75) *$ & $3,22(1,53-6,76)^{*}$ \\
\hline & desconocido & $104(8,81)$ & $1,09(0,86-1,4)$ & $\mathrm{ns}$ & $6(1,40)$ & $0,47(0,19-1,11)$ & $\mathrm{ns}$ \\
\hline & Extrapulmonar (ref.) & $86(7,90)$ & & & $15(2,4)$ & & \\
\hline Localización & Pulmonar & $245(8,44)$ & $1,07(0,83-1,34)$ & $1,48(1,13-1,95)^{*}$ & $34(2,7)$ & $1,1(0,59-2,03)$ & ns \\
\hline & Negativo (ref.) & $49(6,35)$ & & & $5(1,38)$ & & \\
\hline Cultivo & Positivo & $246(9,10)$ & $1,42(1,09-1,83)^{*}$ & ns & $41(3,05)$ & $2,11(0,98-4,54)$ & $2,19(1,00-4,79)^{*}$ \\
\hline & Desconocido & $36(6,96)$ & $0,81(0,56-1,15)$ & ns & $3(1,65)$ & $0,6(0,19-1,96)$ & $\mathrm{ns}$ \\
\hline
\end{tabular}


y 8,7\% en extranjeros en ambos grupos, los casos nacidos fuera de España tuvieron una mayor proporción de traslados que los españoles $(6 \%$ frente a $1,6 \%)$ y de abandonos (3 y $1 \%$ respectivamente). La categoría de "otros, todavía en tratamiento" supuso el $1 \%$ en cada grupo. El 8\% de los casos españoles frente al 3\% de los extranjeros fallecieron durante el tratamiento. Al excluir las muertes del denominador, el porcentaje de RS en los casos españoles aumentó al $88 \%$ frente al $81 \%$ en los extranjeros.

En el estudio de los factores que influyeron en el RPI en los casos españoles, las variables asociadas de forma estadísticamente significativa $(p<0,05)$ en el análisis bivariado fueron: localización pulmonar $(\mathrm{OR}=0,8)$, haber sido tratado previamente $(\mathrm{OR}=2,4)$, o no tener información sobre tratamiento previo $(\mathrm{OR}=1,9)$, confirmación por cultivo $(\mathrm{OR}=0,7)$ o no tener información $(\mathrm{OR}=2,2)$, y ser $\mathrm{VIH}$ positivo( $\mathrm{OR}=1,8)$. En el análisis multivariado desapareció la asociación con la localización extrapulmonar y con la no confirmación por cultivo, permaneciendo el resto (tabla 2). No se observó un riesgo significativo de RPI en ningún grupo de edad.

En los casos nacidos fuera de España, las variables asociadas al RPI en el análisis bivariado fueron: ser hombre $(\mathrm{OR}=1,5)$, tener enfermedad de localización pulmonar $(\mathrm{OR}=1,6)$, no tener información sobre tratamiento previo $(\mathrm{OR}=3,8)$ y ser $\mathrm{VIH}$ positivo $(\mathrm{OR}=1,7)$. Todas estas asociaciones se mantuvieron en el análisis multivariado (tabla 2). Tampoco se encontró relación con la edad.

En cuanto a la mortalidad, las variables que tuvieron asociación estadísticamente significativa en el análisis bivariado en los casos españoles $(\mathrm{p}<0,05)$ fueron: ser hombre $(\mathrm{OR}=1,6)$, confirmación por cultivo( $\mathrm{OR}=1,4)$ y ser mayor de 65 años $(\mathrm{OR}=6,4)$. En el análisis multivariado (tabla 3 ) desapareció la relación con el cultivo y se mantuvo el mayor riesgo para los hombres, además de la localización pulmonar, ser VIH positivo, y también se observó un mayor riesgo de morir a partir de los 45 años, especialmente en los mayores de $65 . \mathrm{La}$ variable tratamiento previo no mostró asociación con la mortalidad.

En los casos extranjeros, las variables asociadas a la mortalidad en el análisis bivariado fueron: ser hombre $(\mathrm{OR}=2,7)$, y VIH positivo $(\mathrm{OR}=3,3)$, y el incremento del riesgo con la edad $(\mathrm{OR}=3$ y 6 en los grupos de 45$64 \mathrm{y}>65$ años). En el análisis multivariado permanecieron los mismos factores de riesgo apareciendo además la confirmación por cultivo. No se observó asociación significativa con la localización de la enfermedad ni con el estatus de tratamiento previo (tabla 3 ).

\section{DISCUSIÓN}

Este estudio es el primero con representatividad nacional sobre los determinantes del tratamiento antituberculoso, constatando que en España el porcentaje de resultados satisfactorios no cumple los objetivos propuestos internacionalmente. También se pone de manifiesto que existen diferencias en cuanto a los resultados del tratamiento y a los factores de riesgo entre los casos españoles y los de origen extranjero. Los españoles tienen mejores resultados de tratamiento pero un porcentaje elevado de fallecimientos mientras que los casos de origen extranjero tienen mayores porcentajes de traslados y de abandonos, lo que pone en evidencia la mayor dificultad en su seguimiento. La coinfección TB-VIH es un factor de riesgo para RPI y mortalidad independientemente del país de nacimiento. Adicionalmente al VIH, los hombres extranjeros con TB pulmonar tienen un mayor riesgo de RPI, y los hombres de edad avanzada mayor riesgo de muerte. En los españoles haber recibido tratamiento previo aumenta el riesgo de RPI y ser hombre, tener TB pulmonar y edad avanzada suponen un mayor riesgo de mortalidad.

En España durante los últimos años se han realizado mejoras en la recogida y actualización de la información sobre resultados de tratamiento en la RENAVE. A pesar de lo anterior, el porcentaje de RS no alcanza el 85\% 
recomendado, ni para el total de los casos ni para los nuevos pulmonares confirmados por cultivo. Esta situación es similar a la de bastantes países europeos, ya que el porcentaje global de RS en la UE/AEE oscila entre un 72 y $76 \%$, sin haber cambiado los últimos años ${ }^{9}$. Esto pone de manifiesto deficiencias en los programas de control de TB, cuyas razones se tienen que investigar. Entre las posibles causas, el ECDC señala problemas en la recogida de información en algunos países, mientras que en otros hay un elevado porcentaje de casos todavía en tratamiento9. Nuestros resultados muestran un porcentaje de casos sin información superior al recomendado, ya que si incluimos a los traslados en el grupo de los que se desconocen los resultados, tal y como hace el ECDC ${ }^{9}$, el porcentaje de casos sin información alcanza el 10\% en los españoles y $15 \%$ en los extranjeros.

Otra explicación del bajo porcentaje de RS en la UE/AEE se debe al elevado número de fallecimientos durante el tratamiento antituberculoso en los países occidentales a costa de los casos nacionales, ya que la edad media en estos es muy superior a la de los casos extranjeros, lo que conlleva mayores complicaciones durante el tratamiento que derivan en muertes ${ }^{4,8,18}$. Esto se pone en evidencia en nuestro estudio, en el que observamos un porcentaje de fallecimientos superior en los casos españoles respecto a los extranjeros. También se observa que si excluimos los fallecimientos del denominador, los casos españoles superan el objetivo del $85 \%$ de RS (88\%), mientras que los de origen extranjero no lo cumplen (81\%). Por el contrario los niños, independientemente de su origen, tienen un porcentaje de RS muy elevado, lo que indica que el seguimiento del tratamiento es muy bueno en España en este grupo de edad.

El peor resultado del tratamiento en los casos de origen extranjero o en los inmigrantes es un hecho que se ha señalado en múltiples estudios, cuya explicación se debe tanto a factores socio-económicos como a epidemiológicos $^{10-12,19-21}$. Se han señalado dificultades en el acceso al sistema sanitario, condiciones de hacinamiento, y dificultades en el seguimiento $^{22,23}$, lo que se evidencia por el elevado porcentaje de traslados en comparación con los casos nacionales ${ }^{8,24,25}$. En España se ha descrito que las personas inmigrantes en situación administrativa irregular o sin cobertura sanitaria tienen un mayor porcentaje de traslados y de pérdidas en el seguimiento ${ }^{24}$, lo que podría deberse en parte a que regresen a sus países de origen. Todo ello requiere una coordinación de los sistemas de vigilancia y de las autoridades sanitarias tanto a nivel nacional como internacional.

Los datos de nuestro estudio muestran que los principales factores de riesgo para el RPI en los casos españoles fueron el tratamiento previo y la coinfección con VIH, ya descritos en otros estudios ${ }^{12,19,26}$. También carecer de información sobre los resultados de cultivo o del estatus de tratamiento se asocia a peores resultados, lo que puede reflejar deficiencias en el seguimiento. Ni el sexo ni la localización de la enfermedad parecen influir en el resultado del tratamiento en los casos nacionales. Tampoco se observa relación entre la edad y el RPI, ni en nacionales ni en extranjeros, coincidiendo con otros estudios ${ }^{11,12,19}$. No obstante, en los casos extranjeros, además de la coinfección con VIH y la falta de información sobre tratamiento previo, se observa que ser hombre y tener localización pulmonar se asocia a malos resultados. Estas dos últimas asociaciones son difíciles de explicar con la información de la que se dispone en la RENAVE y podrían deberse a diversos factores de riesgo que afectan más a los hombres así como otros relacionados con la inmigración, como dificultades en el seguimiento de casos multirresistentes, casos que vuelven a sus países de origen o traslados de los que no se dispone de la conclusión final del tratamiento, ya descritos en otros países ${ }^{25,27}$. En relación a los casos multirresistentes hay que señalar que pueden coexistir varios factores de riesgo, al igual que en países de nuestro entor$\mathrm{no}^{28}$, lo que hace más necesario la mejora en su manejo y seguimiento. En la RENAVE 
todavía no se dispone de información acerca de resistencias de forma individualizada, por lo que algunos de los casos con resultados de tratamiento desconocidos o clasificados como "otros, o todavía en tratamiento", podían corresponder a casos resistentes o multirresistentes de los que no se ha actualizado la información ${ }^{29}$.

En cuanto a la mortalidad, los principales factores de riesgo encontrados, tanto en españoles como en extranjeros (ser hombre, VIH positivo y un aumento de riesgo con la edad) son bien conocidos ${ }^{11,12,18}$. Las únicas diferencias encontradas son la localización pulmonar, que está asociada a un mayor riesgo de muerte en españoles pero no en extranjeros, y la confirmación por cultivo en los extranjeros. Estas dos últimas asociaciones posiblemente se podrían atribuir a causas ajenas a la TB que, de momento, no podemos explicar, ya que no disponemos de información sobre si el fallecimiento se produjo por TB o por otros procesos. Este aspecto se ha tenido en cuenta en los nuevos protocolos en los que la categoría "fallecimiento" se ha desglosado en dos apartados ${ }^{30}$.

Los datos utilizados en este análisis proceden de la RENAVE, que se basa en la declaración de casos, por lo que no se puede descartar que haya cierto grado de subnotificación, dependiendo del funcionamiento de los programas de tuberculosis y de los sistemas de vigilancia en las diferentes CCAA. No obstante, puesto que los resultados obtenidos están en línea con los de otros trabajos, pensamos que este factor no ha afectado sustancialmente a las conclusiones que se pueden derivar de nuestra investigación.

Una de las limitaciones de este estudio es la metodología empleada, lo que dificulta su comparación con otros. Para el cálculo del porcentaje de RS se utilizó el total de casos de la cohorte del año de interés, no sólo los de localización pulmonar confirmados por cultivo tal y como recomienda el ECDC y la OMS ${ }^{9,31}$. Esto se debe a que uno de nuestros objetivos fue evaluar todos los factores que pueden influir en el cumplimiento del tratamiento, por lo que siguiendo estas recomendaciones, hubiéramos excluido los casos extrapulmonares, que representan casi el 30\% del total y cuyo diagnóstico microbiológico es más dificultoso ${ }^{32,33}$. Asimismo, en la categoría de RPI se incluyeron los traslados y a los casos en los que se desconocía el resultado del tratamiento, como en otros estudios realizados a nivel europeo ${ }^{8,29}$, ya que consideramos que no tener información refleja una deficiencia del sistema. La diversidad de criterios a la hora de definir lo que se considera para la TB resultados potencialmente insatisfactorios se pone de manifiesto en una revisión sistemática de distintos estudios realizados en países europeos $^{27}$. Mientras que unos incluían en el grupo de RPI los traslados y las pérdidas en el seguimiento, otros solo consideraron los fallos terapéuticos y abandonos, y algunos excluyeron del cálculo los casos sin información. Esta diversidad de criterios también se pone de manifiesto en los estudios realizados en España ${ }^{10-12,26}$.

Otra limitación es el escaso número de variables o determinantes sociales de los que disponemos a través de los datos nacionales de vigilancia y que ayudarían a entender mejor las causas por las que los casos de origen extranjero tienen peores resultados de tratamiento que los españoles ${ }^{34}$. En los nuevos protocolos aprobados recientemente, se han incluido algunas variables adicionales como las relacionadas con el abuso de drogas y alcohol, personas sin hogar o institucionalizadas $^{30}$. Finalmente, se necesita incorporar la información sobre resistencias a los fármacos antituberculosos en la declaración individualizada, lo que también se tiene en cuenta en los nuevos protocolos. La adaptación a estos cambios nos permitirá caracterizar mejor los grupos de riesgo, lo que constituye una de las necesidades a las que se enfrentan los países de baja incidencia que quieren avanzar hacia el objetivo de la pre-eliminación de la $\mathrm{TB}^{2}$.

Como conclusiones a destacar podemos decir que el porcentaje de resultados satis- 
factorios del tratamiento antituberculoso en España no alcanza los objetivos internacionales, con excepción de los niños, y que existen deficiencias en cuanto a la recogida de información que es necesario subsanar. También hay que señalar que es necesario mejorar el manejo en los casos de TB coinfectados con VIH para disminuir el porcentaje de resultados potencialmente insatisfactorios y de fallecimientos así como prestar atención a los pacientes españoles previamente tratados y a los hombres con TB pulmonar de origen extranjero. El diagnóstico precoz podría disminuir la mortalidad en las personas de edad avanzada. También hay que mejorar el seguimiento y la recogida de información especialmente en los casos extranjeros, para lo cual se necesita una buena coordinación entre las autoridades sanitarias nacionales e internacionales, así como un conocimiento más profundo de los determinantes sociales que afectan a este colectivo.

\section{AGRADECIMIENTOS}

A las personas que trabajan en los Servicios de Epidemiología de las comunidades autónomas, que envían de forma regular la información de los casos de tuberculosis a la Red Nacional de Vigilancia Epidemiológica, y en general a los profesionales de Salud Pública (profesionales de atención primaria y especializada, médicos, personal de enfermería, microbiólogos, etcétera) que contribuyen con su trabajo a mejorar la vigilancia y control de la enfermedad en España.

\section{BIBLIOGRAFÍA}

1. D'Ambrosio L, Dara M, Tadolini M, Centis R, Sotgiu G, van der Werf MJ et al. Tuberculosis elimination: theory and practice in Europe. Eur Respir. J 2014.

2. World Health Organization. Framework for tuberculosis elimination in low-incidence countries. WHO/HTM/ TB/2014.13. 2014. Geneva: WHO; 2014.
3. European Centre for Disease Prevention and Control. Progressing towards TB elimination. A follow-up to the Framework Action Plan to fight tuberculosis in the European Union. Stockholm: ECDC; 2010.

4. European Centre for Disease Prevention and Control (ECDC)/World Health Organization Regional Office for Europe. Tuberculosis surveillance and monitoring in Europe 2014. Stockholm: ECDC;2014.

5. Centro Nacional de Epidemiología. Instituto de Salud Carlos III. Informe epidemiológico sobre la situación de la tuberculosis en España. Año 2012. Madrid:CNE; 2013.

6. Rodriguez-Valin E, Villarubia S, Diaz O. Tuberculosis en España en el año 2013. Situación epidemiológica. Bol Epidemiol Semanal. 2014; 22(15):201-209.

7. Veen J, Raviglione M, Rieder HL, Migliori GB, Graf P, Grzemska M et al. Standardized tuberculosis treatment outcome monitoring in Europe. Recommendations of a Working Group of the World Health Organization (WHO) and the European Region of the International Union Against Tuberculosis and Lung Disease (IUATLD) for uniform reporting by cohort analysis of treatment outcome in tuberculosis patients. Eur Respir J.1998; 12(2):505-510.

8. Manissero D, Hollo V, Huitric E, Kodmon C, AmatoGauci A. Analysis of tuberculosis treatment outcomes in the European Union and European Economic Area: efforts needed towards optimal case management and control. Euro Surveill 2010; 15(11).

9. European Centre for Disease Prevention and Control/WHO Regional Office for Europe.Tuberculosis surveillance and monitoring in Europe 2015. Stockholm: ECDC; 2015.

10. Diez M, et al, Grupo de Trabajo del PMIT-2. Resultados del tratamiento antituberculoso en seis comunidades autónomas españolas. Med Clin (Barc). 2001; 117:474480 .

11. Cayla JA, Caminero JA, Rey R, Lara N, Valles X, Galdos-Tanguis H. Current status of treatment completion and fatality among tuberculosis patients in Spain. Int J Tuberc Lung Dis. 2004; 8(4):458-464.

12. Cayla JA, Rodrigo T, Ruiz-Manzano J, Caminero JA, Vidal R, Garcia JM et al. Tuberculosis treatment adherence and fatality in Spain. Respir Res. 2009; 10:121.

13. Registro Regional de casos de tuberculosis de la Comunidad de Madrid, año 2013. Bol Epidemiol Comunidad de Madrid. 2014; 20(9). 
14. Consellería de Sanidade. Informe de la tuberculosis en Galicia. Características de los casos de tuberculosis de Galicia en el año 2012. Evolución en el periodo 1996-2012. Santiago de Compostela: Xunta de Galicia. Consellería de Sanidade.Dirección Xeral de Innovación e Xestión da Saúde Pública; 2014.

15. Generalitat de Catalunya. Departament de Salut. Informe anual 2013. Situación epidemiológica y tendencia de la endemia tuberculosa en Cataluña. Barcelona: Generalitat de Catalunya. Departament de Salut. Agència de Salut Pública de Catalunya; 2015.

16. Ampliación de la definición de caso de tuberculosis en la Red Nacional de Vigilancia Epidemiológica (I). Bol Epidemiol Sem. 2003; 11(16):181-184.

17. Ampliación de la definición de caso de tuberculosis en la Red Nacional de Vigilancia Epidemiológica (II). Bol Epidemiol Sem. 2003; 11(17):193-195.

18. Lefebvre N, Falzon D. Risk factors for death among tuberculosis cases: analysis of European surveillance data. Eur Respir J. 2008; 31(6):1256-1260.

19. Antoine D, Che D. Treatment outcome monitoring of pulmonary tuberculosis cases notified in France in 2009. Euro Surveill. 2013; 18(12).

20. Mor Z, Kolb H, Lidji M, Migliori G, Leventhal A. Tuberculosis diagnostic delay and therapy outcomes of nonnational migrants in Tel Aviv, 1998-2008. Euro Surveill. 2013; 18(12).

21. Kik SV, Mensen M, Beltman M, Gijsberts M, van Ameijden EJ, Cobelens FG et al. Risk of travelling to the country of origin for tuberculosis among immigrants living in a low-incidence country. Int J Tuberc Lung Dis. 2011; $15(1): 38-43$.

22. Heldal E, Kuyvenhoven JV, Wares F, Migliori GB, Ditiu $\mathrm{L}$, Fernandez dlH et al. Diagnosis and treatment of tuberculosis in undocumented migrants in low- or intermediate-incidence countries. Int J Tuberc Lung Dis. 2008; 12(8):878888.

23. Dara M, de CP, Petrova-Benedict R, Centis R, Zellweger JP, Sandgren A et al. Minimum package for cross-border TB control and care in the WHO European region: a Wolfheze consensus statement. Eur Respir J. 2012; 40(5):1081-1090.

24. Sanz BB, Blasco HT, Galindo CS. Antituberculosis treatment default among the immigrant population: mobility and lack of family support. Gac Sanit. 2009; 23 Suppl $1: 80-85$.

25. Liew SM, Khoo EM, Ho BK, Lee YK, Mimi O, Fazlina MY et al. Tuberculosis in Malaysia: predictors of treatment outcomes in a national registry. Int J Tuberc Lung Dis. 2015; 19(7):764-771.
26. Ruiz-Navarro MD, Espinosa JA, Hernandez MJ, Franco AD, Carrillo CC, Garcia AD et al. [Effects of HIV status and other variables on the outcome of tuberculosis treatment in Spain]. Arch Bronconeumol 2005; 41(7):363-370.

27. Faustini A, Hall AJ, Perucci CA. Tuberculosis treatment outcomes in Europe: a systematic review. Eur Respir J 2005; 26(3):503-510.

28. Anderson LF, Tamne S, Watson JP, Cohen T, Mitnick $\mathrm{C}$, Brown T et al. Treatment outcome of multi-drug resistant tuberculosis in the United Kingdom: retrospectiveprospective cohort study from 2004 to 2007. Euro Surveill 2013; 18(40).

29. Falzon D, Le SY, Belghiti F, Infuso A. Exploring the determinants of treatment success for tuberculosis cases in Europe. Int J Tuberc Lung Dis 2005; 9(11):1224-1229.

30. Grupo de trabajo de los protocolos. Centro Nacional de Epidemiología. Instituto de Salud Carlos III. Red Nacional de Vigilancia Epidemiológica. Protocolos de la Red Nacional de Vigilancia Epidemiológica. Madrid. 2014.

31. World Health Organization. Definitions and reporting framework for tuberculosis - 2013 revision (updated December, 2014). WHO/HTM/TB/2013.2. 2013.

32. Sandgren A, Hollo V, van der Werf MJ. Extrapulmonary tuberculosis in the European Union and European Economic Area, 2002 to 2011. Euro Surveill 2013; 18(12).

33. Solovic I, Jonsson J, Korzeniewska-Kosela M, Chiotan DI, Pace-Asciak A, Slump E et al. Challenges in diagnosing extrapulmonary tuberculosis in the European Union, 2011. Euro Surveill 2013; 18(12).

34. Hargreaves JR, Boccia D, Evans CA, Adato M, Petticrew M, Porter JD. The social determinants of tuberculosis: from evidence to action. Am J Public Health 2011; 101(4):654-662. 